\title{
Atomic energies from the large-dimension limit
}

\author{
J. G. Loeser \\ Department of Chemistry and Society of Fellows, University of Michigan, Ann Arbor, \\ Michigan 48109-1055
}

(Received 23 December 1986; accepted 11 February 1987)

\begin{abstract}
Analytic approximations to nonrelativistic atomic ground state energies are obtained from the first two terms of the $1 / D$ expansion for the $N$-electron atom. These two terms describe the equilibrium structure ( $D \rightarrow \infty$ limit) and normal mode oscillations (1/D term) of a completely symmetric $N$-dimensional configuration of localized particles. The connection between these large- $D$ results and real atoms is established through the vibrational state, which is restricted by antisymmetry requirements at $D=3$. Convergence considerations lead us to consider three different approximations, depending on whether all, none, or part of the results obtained from the $1 / D$ term are used (in addition to those obtained from the $D \rightarrow \infty$ limit); the maximum errors are respectively about $8 \%, 3 \%$, and $1 \%$. In all three approximations the dependence of neutral atom energies on the nuclear charge $Z$ is roughly $Z^{12 / 5}$ for physical $Z$ (as observed for real atoms) and roughly $Z^{7 / 3}$ for very large $Z$ (in agreement with the known asymptotic result). The best approximation, which utilizes the $1 / D$ term up to lowest nonvanishing order in $1 / Z$, is comparable in accuracy to single- $\zeta$ Hartree-Fock calculations.
\end{abstract}

\section{INTRODUCTION}

Dimensional continuation can offer novel conceptual perspectives and computational procedures for a variety of physical problems. The qualitative use of lower-dimensional analogs for real-world problems has long been a familiar procedure. Recently it has been shown, however, that in many cases quantitative predictions for $D=3$ results can also be extracted from $D \neq 3$ calculations. The first and still most striking example of this is the renormalization group approach to critical phenomena, which utilizes $D=4$ as a starting point. ${ }^{1}$

Most recent applications of dimensional continuation have employed the $D \rightarrow \infty$ limit. $^{2}$ This is a classical limit distinct from $h \rightarrow 0$. Often this is augmented by one or more semiclassical correction terms, which together with the $D \rightarrow \infty$ limit constitute the beginning of a $1 / D($ or $1 / N$ ) expansion. ${ }^{3}$ The $1 / D$ expansion has two important characteristics, both arising from the classical nature of the $D \rightarrow \infty$ limit. On the one hand, it is simple. Even for relatively complex systems, like the many-electron atom treated in this paper, it is possible to obtain the leading terms of the $1 / D$ expansion analytically. On the other hand, it is singular. This renders the expansion divergent ( though asymptotic), and accounts for the poor accuracy of results obtained by straightforward application of $1 / D$ perturbation theory.

Substantial improvements in accuracy can be achieved in several ways. First, one can factor out any exactly-soluble component of the energy, such as the hydrogenic component in electronic structure problems, in order to improve the initial convergence of the asymptotic $1 / D$ series. ${ }^{4}$ Second, one can obtain solutions at one or more other values of $D$, such as $D=1$, and combine these with the $1 / D$ expansion. ${ }^{5}$ Finally, one can expand the individual terms of the $1 / D$ series, and discard the divergent contributions. ${ }^{6}$ When applied to two-electron atoms, these procedures are capable of improving the accuracy of the $1 / D$ expansion to the level of conventional electronic structure calculations.

In this paper, we apply the method of $1 / D$ expansions in conjunction with two of the refinements just discussed in order to obtain accurate analytic approximations to the energies of many-electron atoms. First the problem is generalized to spaces to dimensionality $D \neq 3$, and solved for large $D$; explicit expressions for the $D \rightarrow \infty$ limit and $1 / D$ term are obtained. This solution for large $D$ is then recast in a form appropriate for use at small $D$ by utilizing the exact solution for the $D$-dimensional hydrogenic atom. Finally, the energy is expanded in order to reveal the convergence properties of its various components and eliminate those which are divergent. The resulting energy expression yields predictions for total atomic energies which are accurate to about $1 \%$.

The method for treating atoms developed in this paper differs from more familiar methods in several respects. First, it engenders a very different picture of electronic structure. Whereas the starting point in a conventional treatment is one in which the electrons are delocalized and inequivalent (before antisymmetrization), the starting point here is one in which the electrons are completely localized and completely equivalent. The localization is a general consequence of the classical nature of the $D \rightarrow \infty$ limit, while the equivalence is due to the stability of the completely symmetric classical configuration in this particular problem.

A second way in which the dimensional continuation method differs from more familiar ones is in the nature of the approximations which it introduces. In particular, by utilizing the dimensionality of space as its perturbation parameter, the method achieves a zeroth-order solution to the many-body problem without recourse to any averaging or uniformization, as in the Hartree-Fock and Thomas-Fermi methods. This renders the approach especially suitable for the calculation of collective effects like the correlation energy.

A final and especially noteworthy feature of the new method is its extreme simplicity. It involves no parameteri- 
zations, integrations, or iterations, and even the most difficult step, which is to find the roots of a quartic equation, is in principle analytic. In fact, all results reported in this paper can be obtained on a pocket calculator without difficulty. This simplicity is a consequence of the classical character and symmetric geometry of the $D \rightarrow \infty$ limit. It should be noted, however, that although dimensional continuation is much simpler than conventional electronic structure methods, it may not be able to offer comparable precision. In fact, the asymptotic character of $1 / D$ expansions may place a strict limit on the accuracy attainable.

The structure of this paper is as follows. In Sec. II we demonstrate how the many-electron atom can be generalized to $D$-dimensional space, and give explicit expressions for the first two terms of its $1 / D$ expansion. In Sec. III we utilize the exact solution for a one-electron atom of arbitrary dimensionality $D$ and nuclear charge $Z$ to determine the vibrational state and reexpand the energy in the form appropriate for application at small $D$. In Sec. IV we examine convergence of the energy with respect to both $1 / D$ and $1 / Z$ in order to motivate three successively more accurate approximations: $D \rightarrow \infty$ limit plus $1 / D$ term, $D \rightarrow \infty$ limit alone, and $D \rightarrow \infty$ limit plus $1 / D$ term to order $1 / Z$. Finally, in Sec. $V$ we discuss the significance of the results and their possible extension.

\section{SOLUTION FOR LARGE $D$}

Dimensional continuation always involves an extrapolation from known physics, and therefore some degree of arbitrariness. Indeed, any generalization which reduces to known physics at $D=3$ must be considered valid. For the many-electron atom treated here we simply choose the most natural generalization. In the end, however, it is the fact that this generalization leads to a simple and effective computational procedure which serves as its primary justification.

\section{A. Generalization}

Our generalized many-electron atom is defined by a Hamiltonian which is identical to the usual one, except that its Laplacians and Coulomb terms are now interpreted in the manner appropriate to $D$-dimensional Euclidean space. ${ }^{7}$ Thus, for an atom with $N$ electrons and $Z$ protons, we take the Schrödinger equation in atomic units to be

$$
\left[\sum_{i=1}^{N} h_{i}+\sum_{i=1}^{N} \sum_{j=i+1}^{N} g_{i j}\right] \Psi=E \Psi
$$

where

$$
\begin{aligned}
& h_{i}=-\frac{1}{2} \sum_{v=1}^{D} \frac{\partial^{2}}{\partial x_{i v}^{2}}-Z\left[\sum_{v=1}^{D} x_{i v}^{2}\right]^{-1 / 2}, \\
& g_{i j}=\left[\sum_{\nu=1}^{D}\left(x_{i v}-x_{j v}\right)^{2}\right]^{-1 / 2} .
\end{aligned}
$$

We now restrict attention to spherically symmetric states, and recast Eq. (1) in a form convenient for analysis at large $D$. This involves three steps. First we introduce reduced variables, measuring distances in units of $\Omega / Z$ Bohr radii and energies in units of $Z^{2} / \Omega$ hartrees, where $\Omega=\frac{1}{4}(D-1)(D-2 N-1)$. The reason for this choice of scaling will become apparent shortly. Next we transform to internal coordinates, which we take to be the lengths $\rho_{i}$ of the $N$ electron vectors and the cosines $\gamma_{i j}$ of the $\frac{1}{2} N(N-1)$ angles between them. This set of coordinates suffices for $S$ states. Finally, we rewrite the resulting equation as one for the probability amplitude $\Phi=J^{1 / 2} \Psi$, where $J$ is the Jacobian of the transformation to internal coordinates. This eliminates all first derivative terms.

After a bit of work, the details of which will be described elsewhere, ${ }^{8}$ we obtain

$$
(T+U+V) \Phi=\epsilon \Phi,
$$

where the kinetic, centrifugal, and Coulombic terms are

$$
\begin{aligned}
& T=-\frac{1}{2 \Omega} \sum_{i=1}^{N}\left[\frac{\partial^{2}}{\partial \rho_{i}^{2}}+\sum_{j \neq i} \sum_{k \neq i} \frac{\gamma_{j k}-\gamma_{j i} \gamma_{i k}}{\rho_{i}^{2}} \frac{\partial^{2}}{\partial \gamma_{j i} \partial \gamma_{i k}}\right], \\
& U=\frac{1}{2} \sum_{i=1}^{N} \frac{1}{\rho_{i}^{2}} \frac{\Gamma^{(i)}}{\Gamma} \\
& V=-\sum_{i=1}^{N} \frac{1}{\rho_{i}}+\frac{1}{Z} \sum_{i=1}^{N} \sum_{j=i+1}^{N}\left(\rho_{i}^{2}+\rho_{j}^{2}-2 \rho_{i} \rho_{j} \gamma_{i j}\right)^{-1 / 2} .
\end{aligned}
$$

Here, $\Gamma$ is the Gramian ${ }^{9}$ determinant $\left|\hat{\rho}_{i} \cdot \hat{\rho}_{j}\right|=\left|\gamma_{i j}\right|$ for all $N$ electrons, and $\Gamma^{(i)}$ is the Gramian determinant for all but the $i$ th electron. The motivation for scaling distances and energies by $\Omega=\frac{1}{4}(D-1)(D-2 N-1)$ is now apparent. By this choice, the dimensionality $D$ appears as a variable only in the kinetic term $T$, leaving an effective potential $U+V$ which is independent of the dimensionality.

\section{B. Classical limit}

Equation (2) is in a standard form suited for calculations at large $D$. All dimension dependence is confined to the large parameter $\Omega$, which plays the role of an effective mass that localizes the system near the minimum of the effective potential. In the $D \rightarrow \infty$ limit itself the localization is complete, and the solution is given simply by the position and depth of the minimum. This localization (which is quite general) is the reason for calling $D \rightarrow \infty$ a classical limit.

To obtain the minimum of the effective potential, we tentatively assume that it will be a totally symmetric one in which all electrons are equivalent. Because this leaves only two free parameters-a single distance $\rho$ and a single angle $\cos ^{-1} \gamma$-the minimization under this constraint is readily carried out. The solution is

$$
\begin{aligned}
& \rho_{\infty}=\left[\frac{1-\xi / N}{1-\xi}\right]^{2}, \\
& \gamma_{\infty}=\frac{\xi}{\xi-N} \cdot \\
& \epsilon_{\infty}=-\frac{1}{2}\left[\frac{1-\xi}{1-\xi / N}\right]^{3}(N-N \xi+\xi),
\end{aligned}
$$

where $\xi$ is the smallest positive root of the quartic

$$
8 N Z^{2} \xi^{2}(2-\xi)^{2}=(N-\xi)^{3} \text {. }
$$

We will show in the following subsection that the configuration described by Eq. (3) is stable to deformation, and therefore is indeed a local minimum, for all neutral atoms and positive ions. (Not surprisingly, it becomes unstable for 
sufficiently negative ions.) We will not address in this paper the much more difficult question of whether this configuration is in fact the global minimum. To answer this question will probably require a numerical survey of the full $\frac{1}{2} N(N+1)$-dimensional potential energy surface. We note, however, that in the limit of very large atoms, ionization does lower the energy for $N / Z>2^{3 / 2} / 3 \doteq 0.943$, so there will apparently in general be some range of ions for which Eq. (3) describes a local but not a global minimum.

The $D \rightarrow \infty$ limit solution to the $N$-electron atom, as given by $\mathrm{Eq}$. (3), is one in which the electrons are equidistant from one other and equidistant from the nucleus. Thus, the electrons lie at the corners of a regular $N$-point simplex ("hypertetrahedron"), while the nucleus lies along an axis which passes perpendicularly through the center of that figure. For any $N$, the overall geometry is specified by only two parameters, which we have taken to be the reduced distance of the electrons from the nucleus $\left(\rho_{\infty}\right)$ and the angle of the electrons with respect to one another $\left(\cos ^{-1} \gamma_{\infty}\right)$. For neutral atoms, the reduced distance increases monotonically from 1.000 for hydrogen to about 1.547 for large atoms, while the angle decreases monotonically from about $95.3^{\circ}$ for helium to $90.0^{\circ}$ for large atoms. The $D \rightarrow \infty$ limits of the first three atoms $(\mathrm{H}, \mathrm{He}, \mathrm{Li})$ can be pictured in terms of qualitative molecular equivalents $\left(\mathrm{HF}, \mathrm{H}_{2} \mathrm{O}, \mathrm{NH}_{3}\right.$ ). For larger atoms the $D \rightarrow \infty$ limit configuration cannot be embedded in $D=3$, and there are no molecular equivalents.

\section{Normal modes}

For large but finite $D$ the effects of the derivative terms in Eq. (2) must be considered to first order. The rigid classical limit geometry just discussed assumes the role of an equilibrium configuration, and the problem becomes one of determining the normal modes of oscillation about this minimum. The vibrational analysis is greatly simplified by the very high symmetry of the equilibrium configuration, and once again all results can be obtained analytically.

Except for the lightest atoms, which are even simpler, all atoms have just five normal modes. (Hydrogen has one, helium three, and lithium four.) These belong to three different irreducible representation of the symmetric group $S_{N}$, two of which occur twice each. There are first of all two nondegenerate modes belonging to the representation $[N]$, which will be designated $\mathrm{O}^{-}$and $\mathrm{O}^{+}$; these are the totally symmetric stretch and totally symmetric bend, respectively. Then there are two $(N-1)$-fold degenerate modes belonging to [ $N-1$, 1], and designated $\mathbf{1}^{-}$and $\mathbf{1}^{+}$; these are, respectively, stretching and bending modes having single nodal planes. Finally, there is a single $\frac{1}{2} N(N-3)$-fold degenerate mode belonging to $[N-2,2]$, designated simply by 2 ; this mode is purely angular, and is characterized by pairs of nodal planes.

For the simplest atoms, the normal modes can be pictured in terms of their molecular equivalents. For example, the $0^{ \pm}$modes of lithium correspond to the two $A_{1}$ modes of ammonia, and similarly the $1^{ \pm}$modes of the atom to the two $E$ modes of the molecule. The smallest atom with a 2 mode is beryllium, which has no good molecular equivalent. However, because this is a purely angular mode, we can take the $E$ mode of methane as a crude model. (The carbon would need to be displaced along a fourth coordinate axis to provide a better model.)

The normal modes may be found by applying the FG matrix method to Eq. (2). After quite a bit of algebra, ${ }^{8}$ one obtains the five normal mode frequencies (to lowest order in $1 / D)$

$$
\begin{aligned}
& \omega_{0}^{ \pm}=\frac{\Lambda}{D}\left[10-4 \xi+2 \xi^{2} \pm \eta_{0}\right]^{1 / 2}, \\
& \omega_{1}^{ \pm}=\frac{\Lambda}{D}\left[10-18 \xi+7 \xi^{2} \pm \eta_{1}\right]^{1 / 2}, \\
& \omega_{2}=\frac{\Lambda}{D}[16-4(4-3 / N) \xi(2-\xi)]^{1 / 2},
\end{aligned}
$$

where

$$
\begin{aligned}
\Lambda= & {\left[\frac{1-\xi}{1-\xi / N}\right]^{3}, } \\
\eta_{0}= & {\left[132-96 \xi+40 \xi^{2}-16 \xi^{3}\right.} \\
& \left.+4 \xi^{4}-48 \frac{(1-\xi)(2-\xi)}{1-\xi / N}\right]^{1 / 2}, \\
\eta_{1}= & {\left[132-168 \xi+40 \xi^{2}-12 \xi^{3}\right.} \\
& \left.+9 \xi^{4}-24 \frac{(2-3 \xi)(2-\xi)}{1-\xi / N}\right]^{1 / 2} .
\end{aligned}
$$

Note that $\xi$ is still given by Eq. (4).

In general the ordering of the spectrum is given by $\omega_{1}^{-}<\omega_{0}^{-}<\omega_{2}<\omega_{1}^{+}<\omega_{0}^{+}$. Thus, the two predominantly radial modes are of lower frequency than the three predominantly angular modes. For large neutral atoms the five frequencies tend toward the limiting forms

$\begin{array}{ll}\omega_{0}^{-}=\frac{0.83579}{D}, & \omega_{1}^{-}=\frac{0.35422}{D}, \\ \omega_{0}^{+}=\frac{2.07902}{D}, & \omega_{1}^{+}=\frac{1.87530}{D},\end{array}$

The asymmetric stretch mode $1^{-}$becomes very soft for negative ions, and for large atoms vanishes when $N / Z>2^{5 / 2} /$ $5 \doteq 1.131$. This marks the point at which the totally symmetric configuration ceases to be a local minimum.

All of the normal mode frequencies are proportional to $1 / D$ because the effective mass $\Omega$ in Eq. (2b) was asymptotically proportional to $D^{2}$. It will be convenient to make the dimension dependence of the frequencies $\omega$ explicit by expressing them in terms of dimension-independent characteristic values $\lambda$ :

$$
\omega_{0}^{ \pm}=\frac{\lambda_{0}^{ \pm}}{D} \quad \omega_{1}^{ \pm}=\frac{\lambda_{1}^{ \pm}}{D} \quad \omega_{2}=\frac{\lambda_{2}}{D} .
$$

Also for convenience we introduce degeneracy labels for the five representations:

$$
d_{0}^{ \pm}=1 \quad d_{1}^{ \pm}=N-1 \quad d_{2}=\frac{1}{2} N(N-3) .
$$

Finally, we bring together the results of this section. Consider an atom or ion with $n_{\mu}$ quanta in vibrational mode $\mu$ (where $\mu$ runs over $0^{-}, 0^{+}, 1^{-}, 1^{+}$, and 2 ). Combining the results for the classical limit and the normal modes, we ob- 
tain

$$
\epsilon=\epsilon_{\infty}+\frac{\Sigma_{\mu}\left(n_{\mu}+\frac{1}{2} d_{\mu}\right) \lambda_{\mu}}{D} .
$$

Once the vibrational quantum numbers are specified, this gives the total energy for any atom or ion at sufficiently large $D$, correct to order $1 / D$.

\section{APPLICATION TO SMALLD}

In order to apply the results of the preceding section to the real world, we need to determine which vibrational states are consistent with symmetry requirements at low $D$, and how energies vary with dimensionality away from the large$D$ limit. For these purposes the only additional information that will be needed is the exact solution for the energy levels of a hydrogenic atom as a function of $D$. In hartrees, these are given by ${ }^{10}$

$$
E_{n}^{H}=-\frac{1}{2}\left[\frac{2 Z}{D+2 n-3}\right]^{2}
$$

\section{A. Vibrational state}

In our generalization of the $N$-electron atom to $D$-dimensional coordinate space we have tacitly assumed that the spin space and symmetry rules are unaltered. Thus, we require the wave function to be totally antisymmetric at $D=3$. This places certain restrictions on the possible assignments of vibrational quantum numbers $n_{\mu}$. The easiest way to find these is to relate the vibrational states $\left|n_{0}^{-} n_{0}^{+} n_{1}^{-} n_{1}^{+} n_{2}\right\rangle$ to the familiar configurations $\left|n_{1} l_{1} \ldots n_{N} l_{N}\right\rangle$, for which the restrictions imposed by antisymmetry are known. A transcription between the two representations can be obtained in the double limit $D, Z \rightarrow \infty$ where both are valid. (For large $D$, the vibrational description becomes exact, while for large $Z$, single configurations become eigenstates.)

Consider first the $Z \rightarrow \infty$ limit of the vibrational $(D \rightarrow \infty)$ representation. The classical limit solution becomes $\rho_{\infty}=1, \gamma_{\infty}=0, \epsilon_{\infty}=-N / 2$. Furthermore, in the vibrational analysis one finds an exact separation of radial and angular motions, and the limiting characteristic values become $\lambda_{0}^{-}=\lambda_{1}^{-}=2$ (the radial modes) and $\lambda_{0}^{+}$ $=\lambda_{1}{ }^{+}=\lambda_{2}=4$ (the angular modes). Thus, the limiting form for the total energy in Eq. (9) is

$\epsilon=-\frac{N}{2}+\frac{N^{2}+2\left(n_{0}^{-}+n_{1}^{-}\right)+4\left(n_{0}^{+}+n_{1}^{+}+n_{2}\right)}{D}$.

Now consider the corresponding expansion obtained from a pure configuration. For purposes of comparison, it will help to label the contributing orbitals by the quantum numbers $k_{i}$ and $l_{i}$, where $k_{i}=n_{i}-l_{i}-1$ is the number of radial nodes in the $i$ th orbital. $\left(l_{i}\right.$ is, of course, the number of angular nodes.) Upon taking a sum of hydrogenic orbital energies as given by Eq. (10), expressing this in reduced units of $Z^{2} / \Omega$ hartrees, and expanding to order $1 / D$, one finds

$$
\epsilon=-\frac{N}{2}+\frac{N^{2}+2 \sum_{i=1}^{N}\left(k_{i}+l_{i}\right)}{D} .
$$

The quantum numbers in the two representations can now be related in order to reveal the restrictions on vibrational states imposed by antisymmetry. Because of the clean separation of radial and angular motions, two conditions arise:

$$
\begin{aligned}
& n_{0}^{-}+n_{1}^{-}=\sum_{i=1}^{N} k_{i} . \\
& n_{0}^{+}+n_{1}^{+}+n_{2}=\frac{1}{2} \sum_{i=1}^{N} l_{i} .
\end{aligned}
$$

These equations determine a set of candidate vibrational states $\left|n_{0}^{-} n_{0}^{+} n_{1}^{-} n_{1}^{+} n_{2}\right\rangle$ from the (known) set of permissible $S$-state configurations $\left|n_{1} l_{1} \ldots n_{N} l_{N}\right\rangle$. When $Z$ is made finite, one of these candidate states will have lower energy than all others. This will clearly have all quanta assigned to the lowest frequency radial and angular modes, namely $\mathbf{1}^{-}$and 2. Since a totally antisymmetric state can always be constructed from this assignment, it is the vibrational ground state. Note that we limit consideration to the vibrational equivalents of realizable $D=3$ configurations. Thus, we do not consider states like $1 s^{2} 2 s^{2} 2 p^{N-4}$, even though they can be realized for sufficiently large $D$, because such states do not correlate with real-world states.

Although a given vibrational state in general corresponds to more than one configuration (and conversely a given configuration to more than one vibrational state), for the ground state it is not hard to see that the correspondence is one-to-one. Hence we obtain a unique configurational equivalent for the vibrational ground state. We can even go one step further, associating the individual electrons with the orbitals of this configuration. To do this, we consider the atom to be built up one electron at a time. Then each added electron must be accompanied by a certain number of vibrational quanta in order to maintain antisymmetry. By means of Eqs. (13) we can associate these additional quanta with a conventional orbital designator $n_{i} l_{i}$, and call these the shell number and angular momentum of the added electron. This inequivalencing of the symmetrically disposed electrons is so far only conceptual, but we will see in the next subsection that it has consequences for the energetics.

It is instructive to compare the configurational equivalents of $D \rightarrow \infty$ vibrational ground states to known $D=3$ configurations. The two configurations are usually but not always different. For neutral atoms the ordering of orbitals is the same as the familiar one, except that in a roughly degenerate set like $7 s, 6 p, 5 d, 4 f$ (characterized by a common value of $n+l$ ), the ordering is now strictly left to right, rather than approximately right to left. Thus, the transcribed and observed configurations of neutral atoms will differ except for the alkaline earths, which are characterized by the filling of sets like $7 s, 6 p, 5 d, 4 f$. This is illustrated in Fig. 1. We also note that as an atom is ionized, both the transcribed and observed configurations quickly become lexicographic (that is, ordered first by $n$ and second by $l$ ).

\section{B. Reexpansion}

With the vibrational ground state determined, all quantities in the first-order large- $D$ expansion for the energy, Eq. (9), are known. Before this expression can be applied at 

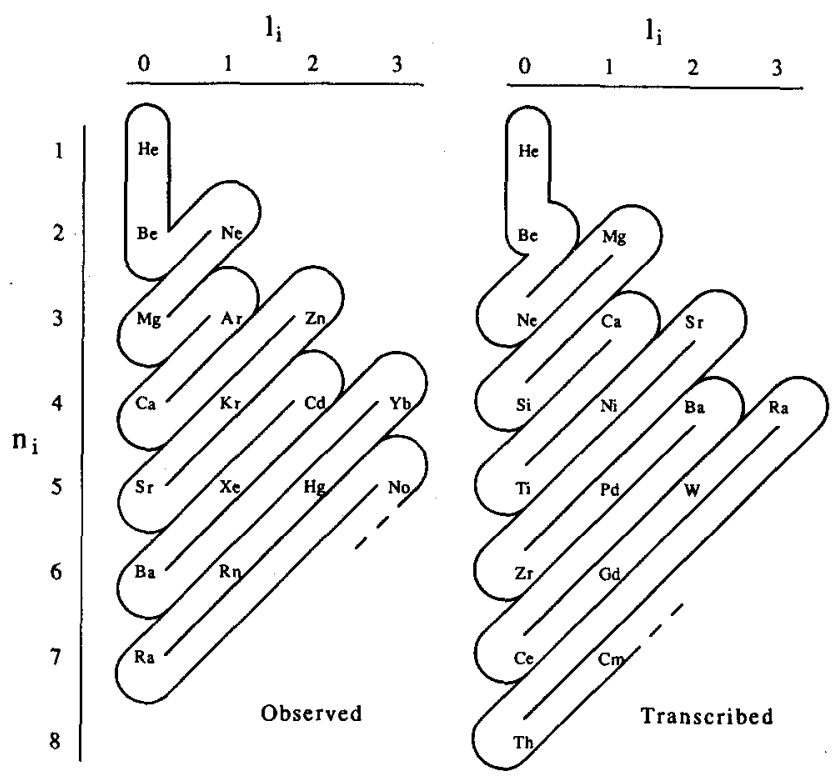

FIG. 1. Periodic structure in real atoms (left) and in the approximations derived from the $D \rightarrow \infty$ limit (right). Elements are grouped according to orbital or vibrational quantum numbers, with only the last element of each group listed. On the left, row and column numbers specify the shell number and angular momentum of the last added electron. On the right they specify the same quantities transcribed via Eqs. (13) from the incremental vibrational quantum numbers. (The ordering in the vibrational representation is actually "normal" up to oxygen, as indicated by the detour to the right of Be.)

$D=3$, however, it must be reexpanded in a form appropriate for small $D$. In its present form the expression gives energies in units of $Z^{2} / \Omega$ hartrees, and $\Omega=\frac{1}{4}(D-1)(D-2 N-1)$ is negative for $D<2 N+1$. Thus, Eq. (9) as is gives no binding for any atom at $D=3$.

The effective mass factor $\Omega$ was chosen to scale energies and distances because this eliminated all dimension dependence from the effective potential. For the purpose of calculating energies, however, what is needed is a factor which eliminates as much dimension dependence as possible from the final energies themselves, especially as we have only calculated contributions to order $1 / D$. Turning again to Eq. (10) for the hydrogenic atom, we see that in the $Z \rightarrow \infty$ limit the factor $\Omega^{\prime}=\frac{1}{4}(D+2 n-3)^{2}$ would eliminate all dimension dependence for electrons in shell $n$. We therefore reexpand Eq. (9) with $Z^{2} / \Omega^{\prime}$ as the overall factor, giving an expression for contributions to the energy from shell $n$ which should behave well at low $D$ as well as high $D$ :

$$
\begin{aligned}
E_{n}(N, Z) & \\
= & {\left[\frac{2 Z}{D+2 n-3}\right]^{2} } \\
& \times\left[\epsilon_{\infty}+\frac{\Sigma_{\mu}\left(n_{\mu}+\frac{1}{2} d_{\mu}\right) \lambda_{\mu}+(2 N+4 n-4) \epsilon_{\infty}}{D}\right] .
\end{aligned}
$$

Note that several quantities in this expression $\left(\epsilon_{\infty}, n_{\mu}, d_{\mu}\right.$, and $\lambda_{\mu}$ ) depend on $N$ and/or $Z$.

As the most natural definition of the total contributions from the various shells we take the successive shell ionization energies. That is, if there are $N_{n}$ electrons with principle quantum numbers less than or equal to $n$ (in either the configurational or vibrational representation), then the total energy attributable to shell $n$ is the difference in energy between ions with $N_{n}$ and $N_{n-1}$ electrons. Upon summing the shell energies, we finally obtain an expression for the total energy (in hartrees) which should be applicable at low D:

$$
E=\sum_{n=1}^{n_{\max }}\left[E_{n}\left(N_{n}, Z\right)-E_{n}\left(N_{n-1}, Z\right)\right] .
$$

It is readily verified that in the $Z \rightarrow \infty$ (hydrogenic) limit which has served as a guide, Eq. (15) always yields the exact energy. The results predicted for finite $Z$ will be presented in the following section.

\section{RESULTS}

We will consider three approximations to the total ground state energies of atoms and ions. The first one is simply that given by Eq. (15). This approximation utilizes the first two terms of the $1 / D$ expansion, and we will call it the 2 term approximation. We then consider two more elementary approximations. One is a much simpler 1-term approximation obtained by dropping all contributions of order $1 / D$; and the other is an intermediate " $1 \frac{1}{2}$-term" approximation in which the $1 / D$ term is included, but only to lowest nonvanishing order in $1 / Z$.

We will see below that the two simpler approximations are in fact more accurate. The reason for this is the singular character of the $1 / D$ perturbation expansion, which causes the $1 / D$ series to diverge except at low order. For the helium atom the divergence is known to set in at third order in $1 / D$, while for larger atoms we will see below that it appears to set in even sooner (though not at lowest order in $1 / Z$ ). These observations will serve to motivate the 1 -term and $1 \frac{1}{2}$-term approximations.

\section{A. 2-term approximation}

The approximations to the total energies of neutral $D=3$ atoms given by Eq. (15) are listed in the first column of Table I. These values pertain to the lowest spherically symmetric vibrational state, as derived in Sec. III. Approximate energies are given for all atoms for which reference values are available in the literature, namely $1 \leqslant Z \leqslant 127$. The reference values, listed in the last column of the table, were obtained from accurate Hartree-Fock-Roothaan calculations ${ }^{11-14}$ corrected for correlation effects. ${ }^{15,16}$

The exact and approximate energies are plotted as a function of atomic number in Fig. 2. Also plotted for comparison are the energies given by the Thomas-Fermi approximation. The overall behavior of the total energies is modeled quite well by the 2-term approximation. Thus, the neutral atom energies are roughly given by $-\frac{1}{2} Z^{12 / 5}$, as observed for real atoms. ${ }^{17,18}$ It is noteworthy that the approximation does not give the $Z^{7 / 3}$-dependence of Thomas-Fermi theory $^{18,19}$ and other simple models. ${ }^{20}$ (In the domain of very large $Z$, which is of course nonphysical for reasons of both nuclear and atomic stability, the energies appear to be proportional to $Z^{7 / 3}$, which is the correct asymptotic nonrelativistic result. ${ }^{21}$ ) 
TABLE I. Nonrelativistic energies (in hartrees) of neutral atoms given by the three approximations of Sec. IV and by accurate conventional calculations."

\begin{tabular}{|c|c|c|c|c|c|}
\hline $\begin{array}{l}\text { Atomic } \\
\text { number }\end{array}$ & Element & $\begin{array}{l}\text { 2-term } \\
\text { approximation }\end{array}$ & $\begin{array}{l}\text { 1-term } \\
\text { approximation }\end{array}$ & $\begin{array}{c}1 \frac{1}{2} \text {-term } \\
\text { approximation }\end{array}$ & $\begin{array}{l}\text { Reference } \\
\text { energies }\end{array}$ \\
\hline 1 & $\mathbf{H}$ & -0.5000000 & -0.5000000 & -0.5000000 & -0.5000000 \\
\hline 2 & $\mathrm{He}$ & -2.9317870 & -2.7377691 & -2.9145458 & -2.9037244 \\
\hline 3 & $\mathbf{L i}$ & -7.4751309 & -7.2653349 & -7.3537232 & -7.4780257 \\
\hline 4 & $\mathrm{Be}$ & -14.942896 & -14.353106 & -14.677196 & -14.666721 \\
\hline 5 & $\mathbf{B}$ & -24.876044 & -24.147777 & -24.356472 & -24.652357 \\
\hline 6 & C & -38.349223 & -37.464031 & -37.464031 & -37.842312 \\
\hline 7 & $\mathbf{N}$ & -55.033685 & -53.931594 & -53.610777 & -54.584524 \\
\hline 8 & O & -76.579754 & -74.578711 & -74.637636 & -75.059270 \\
\hline 9 & F & -101.44654 & -98.994793 & -98.589680 & -99.719300 \\
\hline 10 & $\mathrm{Ne}$ & -131.17621 & -127.32858 & -127.23855 & -128.91905 \\
\hline 11 & $\mathbf{N a}$ & -167.23974 & -161.84479 & -162.33093 & -162.23290 \\
\hline 12 & $\mathbf{M g}$ & -207.37598 & -199.95891 & -200.84280 & -200.02661 \\
\hline 13 & Al & -250.14560 & -242.65214 & -241.74248 & -242.31568 \\
\hline 14 & $\mathbf{S i}$ & -298.41167 & -289.86565 & -287.88059 & -289.32231 \\
\hline 15 & $\mathbf{P}$ & -352.00436 & -342.53053 & -338.79858 & -341.20769 \\
\hline 16 & $\mathbf{S}$ & -411.00889 & -399.66840 & -395.18678 & -398.06085 \\
\hline 17 & $\mathrm{Cl}$ & -477.27732 & -462.54313 & -458.61272 & -460.10187 \\
\hline 18 & $\mathbf{A r}$ & -547.93267 & -530.17038 & -526.17449 & -527.49439 \\
\hline 19 & $\mathbf{K}$ & -628.17242 & -603.80467 & -603.47812 & -599.92453 \\
\hline 20 & $\mathbf{C a}$ & -711.40162 & -682.48623 & -683.13687 & -677.55803 \\
\hline 21 & Sc & -796.15954 & -766.58029 & -764.09561 & -760.57552 \\
\hline 22 & $\mathbf{T i}$ & -887.67777 & -855.96014 & -851.36075 & -849.28575 \\
\hline 23 & $\mathbf{V}$ & -987.24288 & -951.08650 & -945.75796 & -943.80420 \\
\hline 24 & $\mathrm{Cr}$ & -1091.4488 & -1051.5942 & -1045.1571 & -1044.3152 \\
\hline 25 & $\mathrm{Mn}$ & -1206.0183 & -1157.9800 & -1154.3260 & -1150.8657 \\
\hline 26 & $\mathbf{F e}$ & -1323.5607 & -1269.9326 & -1266.5921 & -1263.4832 \\
\hline 27 & Co & -1454.1197 & -1387.8879 & -1391.6201 & -1382.4942 \\
\hline 28 & $\mathrm{Ni}$ & -1585.6805 & -1511.6024 & -1517.4857 & -1507.9905 \\
\hline 29 & $\mathrm{Cu}$ & -1721.2263 & -1651.6132 & -1643.8404 & -1640.1228 \\
\hline 30 & $\mathrm{Zn}$ & -1866.8158 & -1789.0033 & -1782.7655 & -1779.0477 \\
\hline 31 & $\mathrm{Ga}$ & -2017.5368 & -1943.3389 & -1923.6537 & -1924.5004 \\
\hline 32 & $\mathrm{Ge}$ & -2177.7882 & -2094.9729 & -2076.1990 & -2076.6391 \\
\hline 33 & As & -2344.4383 & -2264.1793 & -2232.4103 & -2235.5582 \\
\hline 34 & $\mathrm{Se}$ & -2519.9845 & -2430.6257 & -2399.1361 & -2401.2258 \\
\hline 35 & $\mathrm{Br}$ & -2703.3183 & -2615.2489 & -2571.4603 & -2573.8408 \\
\hline 36 & $\mathbf{K r}$ & -2894.7921 & -2797.0760 & -2752.9272 & -2753.4946 \\
\hline 37 & $\mathbf{R} \mathbf{b}$ & -3083.9497 & -2985.8387 & -2931.9242 & -2939.8270 \\
\hline 38 & $\mathbf{S r}$ & -3303.5986 & -3195.4382 & -3138.9221 & -3133.0579 \\
\hline 39 & $\mathbf{Y}$ & -3508.5648 & -3400.4218 & -3332.4600 & -3333.2312 \\
\hline 40 & $\mathrm{Zr}$ & -3722.8635 & -3612.3010 & -3534.4452 & -3540.5957 \\
\hline 41 & $\mathrm{Nb}$ & -3950.7937 & -3831.1287 & -3747.3899 & -3755.2245 \\
\hline 42 & Mo & -4182.0294 & -4057.2529 & -3965.3189 & -3977.2138 \\
\hline 43 & $\mathrm{Tc}$ & -4429.9079 & -4290.3778 & -4197.5682 & -4206.4953 \\
\hline 44 & $\mathbf{R u}$ & -4678.5054 & -4530.9496 & -4432.0566 & -4443.2864 \\
\hline 45 & $\mathbf{R h}$ & -4947.0474 & -4778.5704 & -4684.4994 & -4687.6833 \\
\hline 46 & Pd & -5213.4985 & -5033.7924 & -4936.1627 & -4939.7471 \\
\hline 47 & $\mathbf{A g}$ & -5476.3838 & -5296.4439 & -5184.5400 & -5199.5652 \\
\hline 48 & $\mathrm{Cd}$ & -5774.2181 & -5573.4539 & -5462.6915 & -5466.9922 \\
\hline 49 & In & -6053.3025 & -5852.0971 & -5726.1814 & -5742.1170 \\
\hline 50 & $\mathrm{Sn}$ & -6373.2872 & -6145.3189 & -6026.2075 & -6024.9220 \\
\hline 51 & $\mathrm{Sb}$ & -6669.0085 & -6440.2738 & -6305.2260 & -6315.5155 \\
\hline 52 & $\mathrm{Te}$ & -7011.9551 & -6750.0143 & -6628.0003 & -6613.8548 \\
\hline 53 & I & -7324.7518 & -7061.6009 & -6922.9636 & -6920.0927 \\
\hline 54 & $\mathrm{Xe}$ & -7691.4742 & -7388.1669 & -7269.3597 & -7234.2902 \\
\hline 55 & Cs & -8021.7853 & -7716.7053 & -7580.6840 & -7556.1286 \\
\hline 56 & $\mathbf{B a}$ & -8413.0986 & -8060.4037 & -7951.5757 & -7885.7787 \\
\hline 57 & $\mathbf{L a}$ & -8761.3633 & -8406.2139 & -8279.6769 & -8223.3412 \\
\hline 58 & $\mathrm{Ce}$ & -9120.7692 & -8759.8497 & -8617.6662 & -8569.2278 \\
\hline 59 & $\operatorname{Pr}$ & -9501.2896 & -9121.0367 & -8972.0038 & -8923.5260 \\
\hline 60 & Nd & -9880.7286 & -9490.6888 & -9328.6316 & -9286.2648 \\
\hline 61 & $\mathrm{Pm}$ & -10284.681 & -9867.9120 & -9705.3433 & -9657.5173 \\
\hline 62 & $\mathrm{Sm}$ & -10684.417 & -10253.721 & -10081.143 & -10037.407 \\
\hline 63 & $\mathbf{E u}$ & -11112.311 & -10647.119 & -10480.976 & -10426.034 \\
\hline 64 & $\mathrm{Gd}$ & -11532.691 & -11049.225 & -10876.481 & -10823.196 \\
\hline 65 & $\mathrm{~Tb}$ & -11944.731 & -11459.685 & -11264.433 & -11229.128 \\
\hline
\end{tabular}


TABLE I. (Continued).

\begin{tabular}{|c|c|c|c|c|c|}
\hline $\begin{array}{l}\text { Atomic } \\
\text { number }\end{array}$ & Element & $\begin{array}{l}\text { 2-term } \\
\text { approximation }\end{array}$ & $\begin{array}{l}\text { 1-term } \\
\text { approximation }\end{array}$ & $\begin{array}{c}11 \text {-term } \\
\text { approximation }\end{array}$ & $\begin{array}{l}\text { Reference } \\
\text { energies }\end{array}$ \\
\hline $\begin{array}{l}66 \\
67 \\
68 \\
69 \\
70\end{array}$ & $\begin{array}{l}\text { Dy } \\
\text { Ho } \\
\text { Er } \\
\mathrm{Tm} \\
\mathrm{Yb}\end{array}$ & $\begin{array}{l}-12417.640 \\
-12847.946 \\
-13347.092 \\
-13796.018 \\
-14322.131\end{array}$ & $\begin{array}{l}-11882.108 \\
-12309.777 \\
-12749.468 \\
-13194.551 \\
-13651.707\end{array}$ & $\begin{array}{l}-11705.988 \\
-12111.126 \\
-12578.750 \\
-13001.438 \\
-13495.931\end{array}$ & $\begin{array}{l}-11644.047 \\
-12067.943 \\
-12500.833 \\
-12942872 \\
-13394.187\end{array}$ \\
\hline $\begin{array}{l}71 \\
72 \\
73 \\
74 \\
75 \\
76 \\
77 \\
78 \\
79 \\
80\end{array}$ & $\begin{array}{l}\mathrm{Lu} \\
\mathrm{Hf} \\
\mathrm{Ta} \\
\mathrm{W} \\
\mathrm{Re} \\
\mathrm{Os} \\
\mathrm{Ir} \\
\mathrm{Pt} \\
\mathrm{Au} \\
\mathrm{Hg}\end{array}$ & $\begin{array}{l}-14790.033 \\
-15343.849 \\
-15831.084 \\
-16413.339 \\
-16920.269 \\
-17464.016 \\
-17990.217 \\
-18556.887 \\
-19102.689 \\
-19692.733\end{array}$ & $\begin{array}{l}-14114.408 \\
-14589.225 \\
-15069.749 \\
-15562.423 \\
-16060.975 \\
-16607.382 \\
-17126.128 \\
-17693.401 \\
-18232.662 \\
-18821.107\end{array}$ & $\begin{array}{l}-13936.535 \\
-14458.697 \\
-14917.581 \\
-15468.212 \\
-15945.741 \\
-16440.946 \\
-16935.879 \\
-17452.797 \\
-17965.407 \\
-18504.524\end{array}$ & $\begin{array}{l}-13854.590 \\
-14324.080 \\
-14802.689 \\
-15290.467 \\
-15787.498 \\
-16293.656 \\
-16809.163 \\
-17334.211 \\
-17868.533 \\
-18412.167\end{array}$ \\
\hline $\begin{array}{l}81 \\
82 \\
83 \\
84 \\
85 \\
86 \\
87 \\
88 \\
89 \\
90\end{array}$ & $\begin{array}{l}\mathrm{Tl} \\
\mathrm{Pb} \\
\mathrm{Bi} \\
\mathrm{Po} \\
\mathrm{At} \\
\mathrm{Rn} \\
\mathrm{Fr} \\
\mathrm{Ra} \\
\mathrm{Ac} \\
\mathrm{Th}\end{array}$ & $\begin{array}{l}-20258.464 \\
-20872.334 \\
-21458.324 \\
-22096.470 \\
-22703.049 \\
-23365.924 \\
-23993.420 \\
-24681.475 \\
-25330.218 \\
-25992.972\end{array}$ & $\begin{array}{l}-19381.205 \\
-19991.128 \\
-20572.383 \\
-21204.090 \\
-21806.822 \\
-22460.620 \\
-23085.151 \\
-23761.345 \\
-24407.995 \\
-25064.004\end{array}$ & $\begin{array}{l}-19035.084 \\
-19596.888 \\
-20145.671 \\
-20730.646 \\
-21297.925 \\
-21906.559 \\
-22492.607 \\
-23125.387 \\
-23730.476 \\
-24347.756\end{array}$ & $\begin{array}{l}-18965.042 \\
-19527.267 \\
-20098.887 \\
-20679.844 \\
-21270.358 \\
-21870.197 \\
-22479.324 \\
-23097.810 \\
-23725.740 \\
-24363.326\end{array}$ \\
\hline $\begin{array}{r}91 \\
92 \\
93 \\
94 \\
95 \\
96 \\
97 \\
98 \\
99 \\
100\end{array}$ & $\begin{array}{l}\mathrm{Pa} \\
\mathrm{U} \\
\mathrm{Np} \\
\mathrm{Pu} \\
\mathrm{Am} \\
\mathrm{Cm} \\
\mathrm{Bk} \\
\mathrm{Cf} \\
\mathrm{Es} \\
\mathrm{Fm}\end{array}$ & $\begin{array}{l}-26689.753 \\
-27377.563 \\
-28103.511 \\
-28816.495 \\
-29571.938 \\
-30310.304 \\
-31029.618 \\
-31857.947 \\
-32599.160 \\
-33459.834\end{array}$ & $\begin{array}{l}-25728.538 \\
-26403.599 \\
-27087.181 \\
-27781.399 \\
-28484.130 \\
-29197.607 \\
-29921.121 \\
-30655.485 \\
-31398.984 \\
-32153.294\end{array}$ & $\begin{array}{l}-24989.795 \\
-25629.929 \\
-26299.285 \\
-26962.745 \\
-27660.059 \\
-28347.317 \\
-29017.376 \\
-29782.172 \\
-30472.755 \\
-31269.509\end{array}$ & $\begin{array}{l}-25010.820 \\
-25668.112 \\
-26335.02 \\
-27012.26 \\
-27699.30 \\
-28396.24 \\
-29103.78 \\
-29821.22 \\
-30548.86 \\
-31286.70\end{array}$ \\
\hline $\begin{array}{l}101 \\
102 \\
103 \\
104 \\
105 \\
106 \\
107 \\
108 \\
109 \\
110\end{array}$ & $\begin{array}{l}\text { Md } \\
\text { No } \\
\text { Lw }\end{array}$ & $\begin{array}{l}-34223.227 \\
-35116.875 \\
-35902.737 \\
-36829.995 \\
-37638.621 \\
-38600.129 \\
-39431.817 \\
-40360.805 \\
-41214.747 \\
-42174.437\end{array}$ & $\begin{array}{l}-32916.919 \\
-33691.312 \\
-34475.206 \\
-35269.818 \\
-36074.122 \\
-36889.091 \\
-37713.947 \\
-38573.600 \\
-39420.444 \\
-40302.316\end{array}$ & $\begin{array}{l}-31980.946 \\
-32810.371 \\
-33542.993 \\
-34405.804 \\
-35159.941 \\
-36056.852 \\
-36832.834 \\
-37687.654 \\
-38483.951 \\
-39369.033\end{array}$ & $\begin{array}{l}-32034.94 \\
-32793.58 \\
-33562.12 \\
-34340.76 \\
-35129.70 \\
-35928.94 \\
-36738.48 \\
-37558.32 \\
-38.388 .66 \\
-39229.40\end{array}$ \\
\hline $\begin{array}{l}111 \\
112 \\
113 \\
114 \\
115 \\
116 \\
117 \\
118 \\
119 \\
120\end{array}$ & & $\begin{array}{l}-43050.912 \\
-44041.824 \\
-44941.114 \\
-45963.770 \\
-46886.154 \\
-47941.075 \\
-48886.835 \\
-49974.544 \\
-50943.959 \\
-52064.977\end{array}$ & $\begin{array}{l}-41171.354 \\
-42075.641 \\
-42967.078 \\
-43893.975 \\
-44808.016 \\
-45757.720 \\
-46694.570 \\
-47667.277 \\
-48627.141 \\
-49623.048\end{array}$ & $\begin{array}{l}-40185.902 \\
-41101.816 \\
-41939.510 \\
-42886.828 \\
-43745.603 \\
-44724.894 \\
-45605.006 \\
-46616.840 \\
-47518.543 \\
-48563.491\end{array}$ & $\begin{array}{l}-40080.54 \\
-40942.28 \\
-41814.02 \\
-42696.26 \\
-43588.80 \\
-44491.74 \\
-45405.18 \\
-46329.12 \\
-47263.26 \\
-48207.70\end{array}$ \\
\hline $\begin{array}{l}121 \\
122 \\
123 \\
124 \\
125 \\
126 \\
127\end{array}$ & & $\begin{array}{l}-53058.330 \\
-54067.926 \\
-55127.622 \\
-56166.288 \\
-57259.600 \\
-58327.357 \\
-59454.483\end{array}$ & $\begin{array}{l}-50606.130 \\
-51599.583 \\
-52602.124 \\
-53616.618 \\
-54640.184 \\
-55675.798 \\
-56720.466\end{array}$ & $\begin{array}{l}-49487.041 \\
-50424.518 \\
-51397.789 \\
-52361.403 \\
-53365.696 \\
-54355.871 \\
-55391.747\end{array}$ & $\begin{array}{l}-50124.69 \\
-51101.64 \\
-52089.68 \\
-53088.82 \\
-54099.18 \\
-55120.71\end{array}$ \\
\hline
\end{tabular}

a Reference energies were obtained from Hartree-Fock calculations of Refs. 11-14 and correlation energy estimates of Refs. 15-16. 


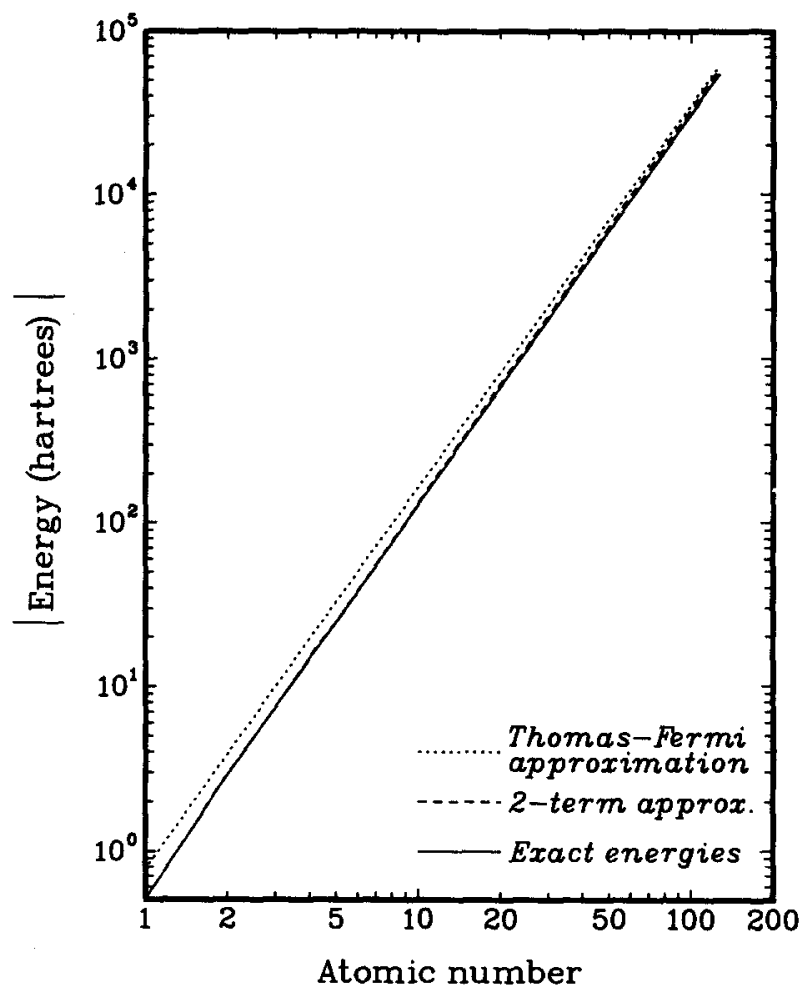

FIG. 2. Nonrelativistic energies of neutral atoms $(1 \leqslant Z \leqslant 127)$ given by the 2-term approximation [Eq. (15), dashed line], by accurate numerical calculations (Refs. 11-16, solid line), and by the Thomas-Fermi approximation (Ref. 19, dotted line). Not plotted are the 1-term and $1 \frac{1}{2}$-term approximations, both of which would fall within a pen width of the exact values. Overall, the 2-term and exact energies are given roughly by $-0.53 Z^{12 / 5}$ and the Thomas-Fermi energies by $-0.77 Z^{7 / 3}$.

The top curve of Fig. 3 gives the percent errors in the 2term energies relative to the exact values. The approximation overestimates the binding energies for all atoms except lithium, and the error increases with atomic number. Thus, for $Z \leqslant 10$ the error is $2 \%$ or less, while for large atoms it can be as large as $8 \%$. This level of accuracy is comparable to that achieved in variational calculations with several parameters (e.g., 2 per $l$ value ${ }^{20}$ ). The present approximation is much simpler, but not variational.

Superimposed on the overall trend of energies is a kind of fine structure, which is quite apparent in the error plot. This structure arises from the changing vibrational state, the larger local maxima marking the points at which the "valence" vibrational level has been assigned as many quanta as possible (so that a new level is excited in the next atom). The fine structure is not very realistic, though it is of the same order of magnitude as that found in real atoms. This is illustrated in Fig. 4, where the dominant power-law components of the exact and approximate energies (straight lines in Fig. 2) are factored out to reveal the small irregularities with respect to $Z$.

Although the present approximation utilizes only two terms of the $1 / D$ expansion, it is already necessary to consider the question of convergence. Quite generally $1 / D$ expansions are asymptotic, and diverge at high order. ${ }^{22}$ In fact, for most systems studied to date, the divergence sets in already

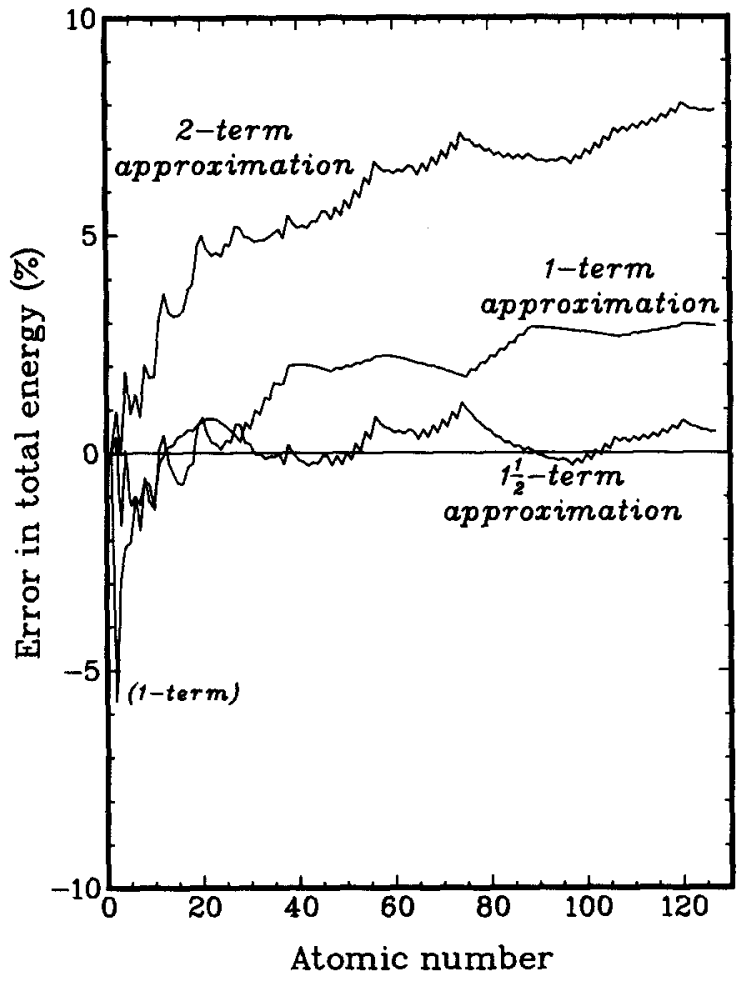

FIG. 3. Errors in the neutral atom energies $(1 \leqslant Z \leqslant 127)$ given by the three approximations derived from the $1 / D$ expansion. Percent errors were calculated relative to the sum of calculated Hartree-Fock energies and estimated correlation energies. Hartree-Fock energies were obtained from Refs. 11 $(2 \leqslant Z \leqslant 54), 12(55 \leqslant Z \leqslant 92), 13(93 \leqslant Z \leqslant 120)$, and $14(122 \leqslant Z \leqslant 127)$; correlation energies were obtained from Refs. $15 \quad(2 \leqslant Z \leqslant 18)$ and 16 $(19 \leqslant Z \leqslant 127)$.

at low order when $D=3$. In the helium atom, for example, the perturbation contributions reach a minimum at second order, and grow rapidly thereafter. ${ }^{5}$

Typically in an asymptotic series the error in a given partial sum is bounded at least roughly by the magnitude of the next term in the series. The optimal approximation is therefore obtained by truncating the series just before the smallest term. ${ }^{23}$ Thus, for the helium atom the best approximation is the sum of the zeroth- and first-order terms of the $1 / D$ expansion-the 2-term approximation.

For larger neutral atoms it appears that the convergence is no better than in helium, and may be worse. The evidence is indirect, because the only available data are the first two terms of the $1 / D$ expansion and the remainder term, obtained by subtracting these from accurate nonrelativistic energies. (If the $1 / D$ series converged, this would of course be the sum of the higher-order terms.) These three contributions to the energy are plotted for all atoms studied in Fig. 5 .

For small atoms $(Z \leqslant 12)$ the first-order term is larger than the error term. This is consistent with the use of a 2term approximation, as in helium. For larger atoms $(Z>12)$, however, the first-order term is smaller than the error, which suggests that it is smaller than the second-order term. Thus, it would apparently be better to truncate the series before rather than after the $1 / D$ term. We now turn to this much simpler approximation. 


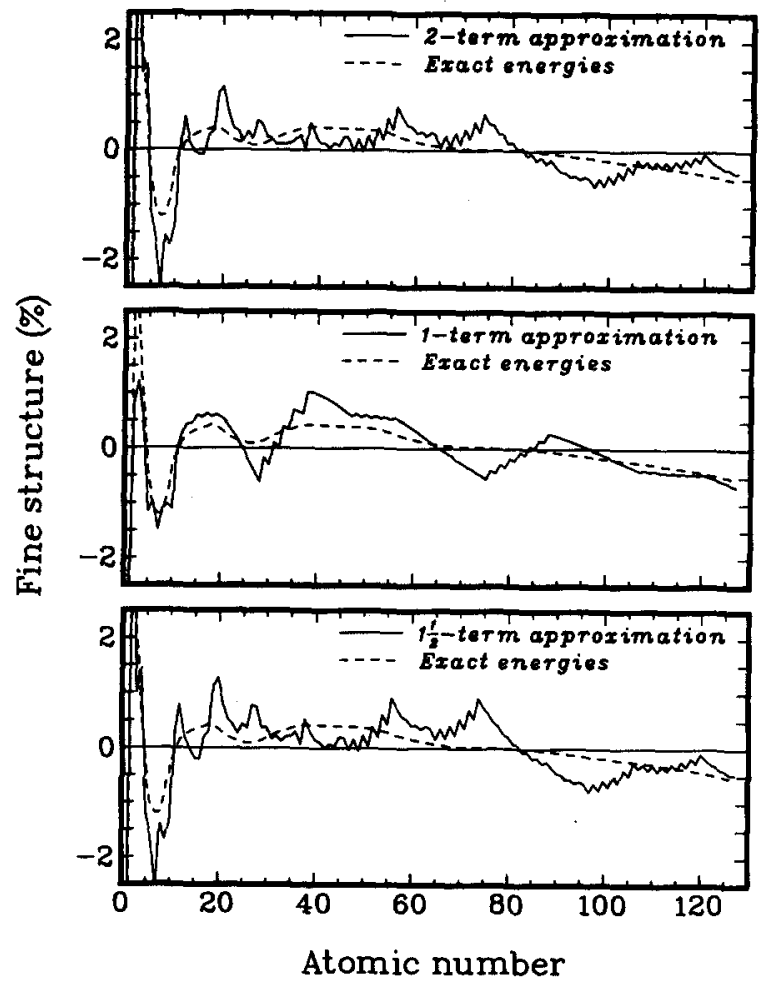

FIG. 4. Fine structure with respect to $Z$ in the exact and approximate energies of neutral atoms. For each set of energies the fine structure is calculated as the percent deviation relative to the best fit by a power law $a Z^{\alpha}$ (i.e., by a straight line in Fig. 2) to that set of energies. For the exact energies and the three approximations (2-term, 1-term, and 11 -term) these fits were, respectively, $(a, \alpha)=(-0.534,2.384),(-0.526,2.403),(-0.515,2.398)$, and $(-0.529,2.387)$.

\section{B. 1-term approximation}

For the 1-term approximation all contributions of order $1 / D$ in Eq. (14) are dropped, leaving only the $D \rightarrow \infty$ limit term $\epsilon_{\infty}$. Thus, the 1-term approximation is given explicitly by

$$
E=\sum_{n=1}^{n_{\max }}\left[\frac{2 Z}{D+2 n-3}\right]^{2}\left[\epsilon_{\infty}\left(N_{n}, Z\right)-\epsilon_{\infty}\left(N_{n-1}, Z\right)\right] .
$$

Note that although no contributions of order $1 / D$ are included, the $1 / D$ term is still used to determine the vibrational shell structure. (To obtain an approximation based solely on the $D \rightarrow \infty$ limit, it would be necessary to assign the vibrational state without reference to the vibrational frequencies. This can be done in several ways, but we will not discuss the results here.)

The total energies given by the 1-term approximation for neutral atoms are given in the second column of Table I. These energies would fall within a pen width of the reference energies in Fig. 2, and are therefore not plotted there. The percent errors with respect to the exact values are plotted as the middle curve of Fig. 3. The results are completely consistent with the suggestion that it would be better to drop the 1/ $D$ term except for the smallest atoms. In fact, the 1-term approximation is better than the 2-term for all atoms with $Z \geqslant 8$. The binding energies are still in general overestimated,

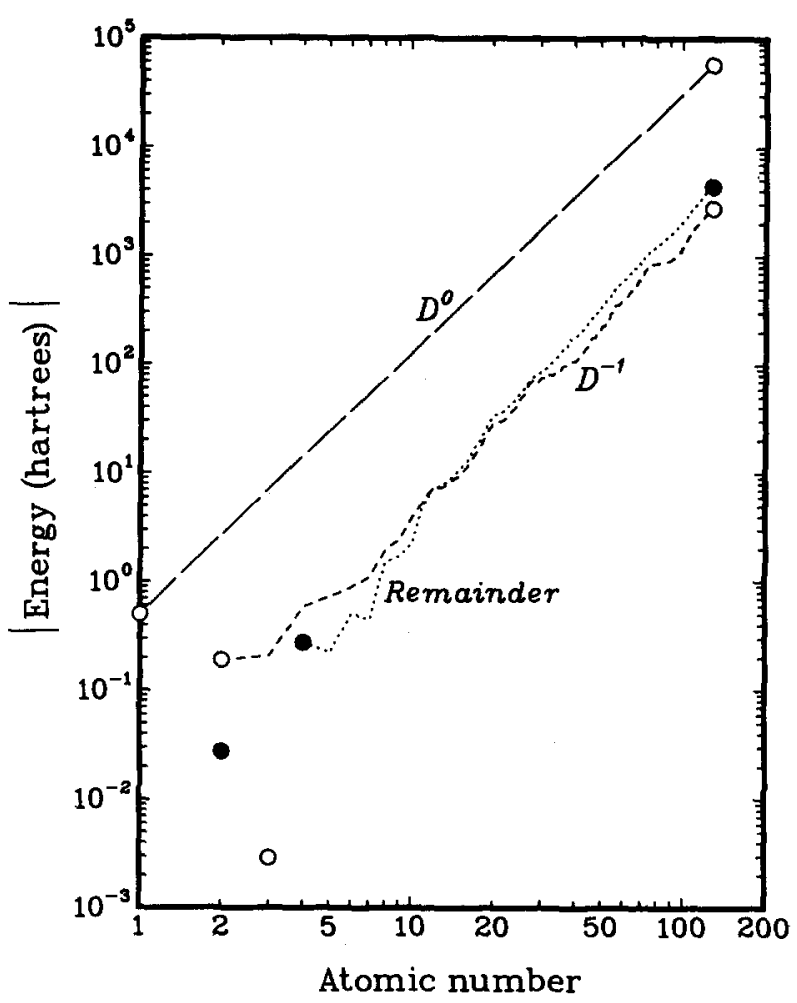

FIG. 5. Breakdown of total atomic energies with respect to $1 / D$. Filled and open circles at the ends of the curves designate positive and negative values, respectively. The zeroth-order contribution $\left(D^{\circ}\right)$ by itself is Eq. (16), the 1term approximation; adding the first-order contribution $\left(D^{-1}\right)$ gives $\mathrm{Eq}$. (15), the 2-term approximation. The remainder term was calculated as the difference between accurate reference energies and the 2-term approximation.

but now by at most about $3 \%$. For $Z \leqslant 12$ the energies are underestimated.

Asymptotically the neutral atom energies given by the 1-term approximation can be shown to behave as $Z^{7 / 3}$, in agreement with Thomas-Fermi theory. However, for the known elements the behavior is again much closer to $Z^{12 / 5}$, as observed for real atoms. (In fact, although the ThomasFermi result is the correct limiting form, it is worse than the 1-term approximation for $Z<5000$.)

With the $1 / D$ term gone, the "fine structure" superimposed on the overall trend of energies is more tempered and in some ways more realistic. As shown in Fig. 4, most of the irregularities in the curve of reference energies are present in the 1-term approximation, though in a somewhat caricatured form, with exaggerated and sharpened figuration. It is interesting to note that for most atoms the local features predicted by the 1-term and 2-term approximations fall on opposite sides of those present in the true energies. We have no explanation for this, but remark that the partial sums of $1 / D$ series have been observed to oscillate about the correct results in other contexts as well..$^{24,25}$

The 1-term approximation is superior to the 2-term approximation on several counts, including simplicity, overall accuracy, and fine structure. Except for the smallest atoms, the $1 / D$ term does not help. As discussed above, this is apparently because the asymptotic $1 / D$ series has already begun to diverge by first order. We now turn to consider how a 


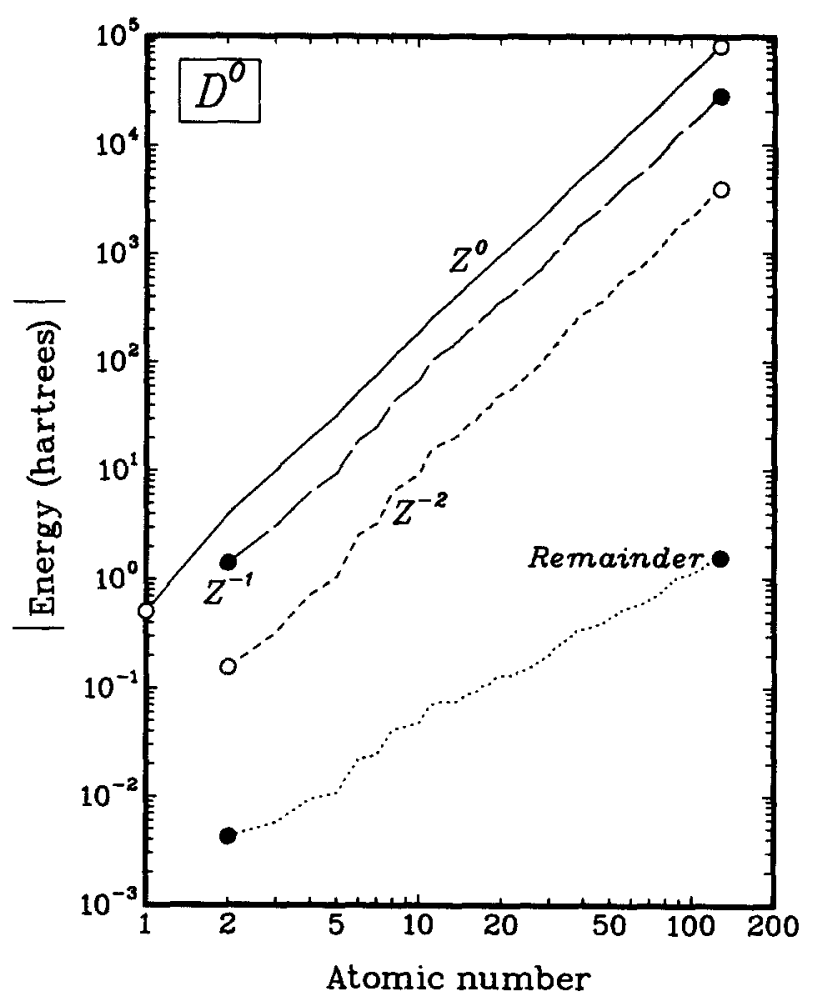

FIG. 6. Breakdown of the zeroth-order energy with respect to $1 / Z$. Filled and open circles at the ends of the curves denote positive and negative values, respectively. Curves were obtained by evaluating the 1-term approximation, Eq. (16), at successive orders of $1 / Z$ [using Eq. (17) and the infinite-order result of $\mathrm{Eq}$. (3c) ].

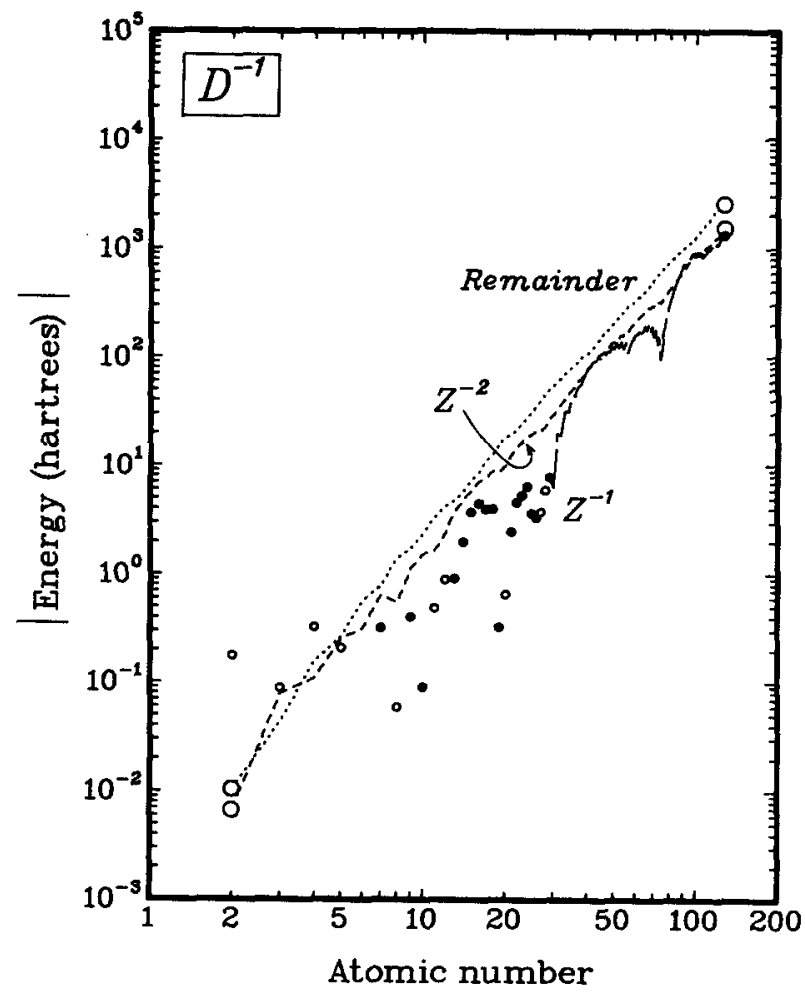

FIG. 7. Breakdown of the first-order energy with respect to $1 / Z$. Filled and open circles again denote positive and negative values, respectively. The contribution of order $1 / Z$ changes sign often for elements below copper. Curves were obtained by evaluating the difference between the 2-term and 1-term approximations, Eq. (15) and Eq. (16), at successive orders of $1 / Z$. convergent component can be extracted from the $1 / D$ term. By putting this back in, we obtain an approximation intermediate between the 1-term and 2-term approximations in complexity, yet superior to both in accuracy.

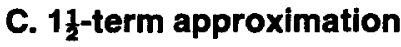

Although there might be other ways to partition the individual terms of the $1 / D$ expansion, the most natural way is to expand each of them in powers of $1 / Z$. As Eq. (2) makes clear, this is just a perturbation expansion in the interelectron repulsion, starting with the hydrogenic or independent-electron solution at zeroth order.

In Figs. 6 and 7, the first two terms of the $1 / D$ expansion are partitioned in this way. In each case the contributions through order $1 / Z^{2}$ are plotted along with the remainder term. (In this case the remainder term is the sum of the higher-order contributions, because the $1 / Z$ series converge.) The perturbation expansions were obtained by expanding the solution to Eq. (4) and then using this in Eqs. (3) and (5), giving

$$
\begin{gathered}
\epsilon_{\infty}=-\frac{N}{2}\left[1-\frac{N-1}{2^{1 / 2} Z}+\frac{(N-1)(4 N-3)}{32 Z^{2}}-\cdots\right], \\
\lambda_{0}^{-}=2-\frac{N-1}{2^{-1 / 2} Z}+\frac{(N-1)(24 N-25)}{96 Z^{2}}-\cdots, \\
\lambda_{0}^{+}=4-\frac{12 N-11}{2^{5 / 2} Z}+\frac{144 N^{2}-176 N+65}{768 Z^{2}}-\cdots, \\
\lambda_{1}^{-}=2-\frac{11 N-8}{2^{5 / 2} Z}+\frac{141 N^{2}-218 N+100}{384 Z^{2}}-\cdots, \\
\lambda_{1}=4-\frac{14 N-11}{2^{5 / 2} Z}+\frac{264 N^{2}-256 N+65}{768 Z^{2}}-\cdots, \\
\lambda_{2}=4-\frac{16 N-15}{2^{5 / 2} Z}+\frac{128 N^{2}-192 N+75}{256 Z^{2}}-\cdots
\end{gathered}
$$

Figures 6 and 7 reveal that the $1 / Z$ perturbation expansion is much more convergent for the zeroth-order term than for the first-order term. By itself the slower convergence of the first-order term (Fig. 7) is not a problem, since we know the result to infinite order. (This was how the error term was obtained.) However, comparing Figs. 6 and 7, one sees that convergence with respect to $1 / D$ is affected. The $1 / D$ series, which cannot be taken to infinite order, is apparently better behaved at low order in $1 / Z$ than at high order. This suggests that the low-order contributions to the $1 / D$ term might help.

The simplest possible approximation of this form, which we will call the $1 \frac{1}{2}$-term approximation, is obtained by including the $1 / D$ term to lowest nonvanishing order in $1 / Z$, namely first order. (The contribution of zeroth order in $1 / Z$ vanishes identically because this is just the $Z \rightarrow \infty$ limit uti- 
lized in Sec. III to reexpand the energy.) This approximation would be justified if at this order of $1 / Z$, but not at higher order, the $1 / D$ term were still in the convergent regime. For the two-electron atom this is indeed the case, ${ }^{25}$ but for larger atoms it is only conjecture.

If we assume that only the $1^{-}$and 2 modes are excited, as in the vibrational ground state, then the $1 \frac{1}{2}$-term approximation reduces to

$$
E=\left.\sum_{n=1}^{n_{\max }}\left[\frac{2 Z}{D+2 n-3}\right]^{2}\left[\epsilon_{\infty}+\frac{(32 n-35) N^{2}-\left(44 n_{1}^{-}+64 n_{2}-3\right) N-4 n_{2}}{2^{9 / 2} D Z}\right]\right|_{N_{n-1}} ^{N_{n}}
$$

Again we note that $n_{1}^{-}$and $n_{2}$, which count the number of radial and angular nodes, vary with $N$.

The total energies given by the $1 \frac{1}{2}$-term approximation are listed in the third column of Table I, and the errors relative to accurate energies are plotted as the bottom curve of Fig. 3. The new approximation, which is intermediate between the other two in complexity, is the most accurate of the three. The errors are less than $1 \%$ for almost all atoms. This is similar to the accuracy achieved in single- $\zeta$ HartreeFock calculations. ${ }^{11}$ The binding energies are still usually overestimated, though there are now stretches throughout the known Periodic Table where they are underestimated.

Although the overall accuracy of the energies is much improved in the $1 \frac{1}{2}$-term approximation, the fine structure is once again more pronounced and less realistic. In fact, Fig. 4 reveals that it is almost identical to that of the 2-term approximation. Thus, the lowest-order contribution to the $1 / D$ term (the " $\frac{2}{2}$ " term) helps the overall energies, but not the fine structure. On the other hand, the higher-order contributions (the remaining " $\frac{1}{2}$ ") hurt the overall energies, but have almost no effect on the fine structure.

The $1 \frac{1}{2}$-term approximation is about as good as one can do using only the first two terms of the $1 / D$ expansion. There is one further variant that might be considered, namely one in which the $1 / Z$ expansion for the zeroth-order term is also truncated. From Figs. 6 and 7 one sees that this would apparently be justified after order $1 / Z^{2}$, where the first-order contributions exceed the zeroth-order. However, one also sees that the truncation would have almost no effect, since the terms dropped (the remainder term in Fig. 6) are so small anyway. Thus, to improve significantly on the $1 \frac{1}{2}$-term approximation it will be necessary to go to higher order in $1 / D$. As we will see in the next section, there is a good chance that this will in fact help.

\section{SUMMARY AND PROSPECTS}

We have introduced three methods based on the $1 / D$ expansion for obtaining analytic approximations to atomic energies. All three methods utilize the zeroth-order term of the expansion, but they differ in the extent to which they utilize the first-order term. The latter is used either in its entirety (the 2-term approximation), to lowest nonvanishing order in the interelectron repulsion (the $1 \frac{1}{2}$-term approximation), or only to obtain the quantum numbers (the 1term approximation). In each case the resulting expression for the energy at large $D$ is recast in a form appropriate for application at $D=3$ by recognizing how the energy of a hy- drogenic atom varies with quantum number and dimensionality.

The physical content of the terms entering the approximations is unconventional, though in some ways it is reminiscent of the concepts of the old quantum theory. The zeroth-order $(D \rightarrow \infty)$ solution is a classical one in which the electrons are completely localized relative to one another and to the nucleus. It also happens to be highly symmetric, since the electrons assume positions which are completely equivalent. The first-order $(1 / D)$ term, on the other hand, is a semiclassical correction for the effects of large but finite $D$. It describes normal mode vibrations about the symmetric equilibrium geometry.

This picture of electronic structure is of course radically different from the usual orbital description, stressing complementary aspects of the solution to the problem. Thus, although dimensional continuation may not be able to produce good ionization energies or term structures, it does promise to model collective effects like the correlation energy very well. ${ }^{6,26}$ This is because these effects, which are so difficult to model by conventional methods, are already included at zeroth order in the new approach. Even at this order, one obtains atomic correlation energies that agree to within the estimated uncertainties with most known values. ${ }^{27}$ The new method also gives insight into the dynamics of electron correlation, ${ }^{28}$ and helps to explain the success of certain other approaches (such as that of hyperspherical coordinates ${ }^{29}$ ) to the correlation problem.

These observations suggest that dimensional continuation might best be used in conjunction with conventional approaches. For example, the Hartree-Fock method could be utilized to calculate approximate energies or properties, and then a low-order $1 / D$ expansion to calculate correlation corrections. ${ }^{6}$ It is quite possible, however, that dimensional continuation methods can be improved to the point where they will give accurate results on their own.

The most obvious way to improve upon the results discussed in this paper would be to calculate higher-order terms in $1 / D$. These will probably best be truncated, like the firstorder term. Indeed, it appears quite likely that the full $1 / D$ series will diverge beyond first order. The contributions of lowest order in $1 / Z$, however, may not diverge until higher order. For two-electron atoms, for example, these truncated contributions converge through sixth order in $1 / D, 4$ even though the full $1 / D$ series converges only through second order. $^{5}$

We note that the attempt to calculate higher-order terms in the $1 / D$ expansion might be facilitated greatly by 
two approximations. First, it would probably suffice to calculate the terms only to lowest nonvanishing order in $1 / Z$, since they would most likely need to be truncated at that order anyway. Second, since the correlation energy is modeled so well by lower-order calculations, and is in any case only a small fraction of the energy in larger atoms, the higher-order terms could be calculated in the Hartree-Fock approximation.

If by means of either combination with standard methods or calculation to higher order it does prove possible to calculate atomic energies to chemical accuracy, then it would be natural to try to apply the same method to molecules. The generalization of molecular Hamiltonians to arbitrary dimensionality and their analysis at large $D$ will probably require significant work, but there is no apparent reason why the methods described in this paper should not work for molecules as well.

\section{ACKNOWLEDGMENT}

This work would not have been possible without the foundations laid by Dudley Herschbach, who first recognized the full potential for dimensional continuation as applied to electronic structure, and introduced me to the subject.

'K. G. Wilson, Rev. Mod. Phys. 55, 583 (1983).

${ }^{2}$ L. G. Yaffe, Phys. Today 36 (No. 8), 50 (1983); Rev. Mod. Phys. 54, 407
(1982).

${ }^{3}$ E. Witten, Phys. Today 33 (No. 7), 38 (1980).

${ }^{4}$ D. R. Herschbach, J. Chem. Phys. 84, 838 (1986).

5J. G. Loeser and D. R. Herschbach, J. Chem. Phys. 86, 2114 (1987).

${ }^{6}$ J. G. Loeser and D. R. Herschbach, J. Chem. Phys. 86, 3512 (1987).

${ }^{7}$ J. G. Loeser and D. R. Herschbach, J. Chem. Phys. 84, 3882, 3893 (1986).

${ }^{8}$ J. G. Loeser, J. Chem. Phys. (to be submitted).

${ }^{9}$ F. R. Gantmacher, The Theory of Matrices (Chelsea, New York, 1959), Vol. 1, p. 247.

${ }^{10}$ D. R. Herrick and F. H. Stillinger, Phys. Rev. A 11, 42 (1975).

"E. Clementi and C. Roetti, At. Data Nucl. Data Tables 14, 177 (1974).

${ }^{12}$ A. D. McLean and R. S. McLean, At. Data Nucl. Data Tables 26, 197 (1981).

${ }^{13}$ J. P. Desclaux, At. Data Nucl. Data Tables 12, 311 (1973).

${ }^{14}$ A. C. Larson and J. T. Waber, J. Chem. Phys. 48, 5021 (1968),

${ }^{15}$ A. Veillard and E. Clementi, J. Chem. Phys. 49, 2415 (1968).

${ }^{16}$ R. D. Cowan, The Theory of Atomic Structure and Spectra (Univ. of California, Berkeley, 1981 ), p. 203.

${ }^{17}$ L. L. Foldy, Phys. Rev. 83, 397 (1951).

${ }^{18} \mathrm{~J}$. M. C. Scott, Philos. Mag. 43, 859 (1952).

${ }^{19}$ E. A. Milne, Proc. Cambridge Philos. Soc. 23, 794 (1927).

${ }^{20}$ W. E. Thirring, A Course in Mathematical Physics (Springer, New York, 1979), Vol. 3, pp. 6 and 265.

${ }^{21}$ E. H. Lieb and B. Simon, Adv. Math. 23, 22 (1977).

${ }^{22}$ D. J. Doren and D. R. Herschbach, Phys. Rev. A 34, 2654 (1986),

${ }^{23}$ C. M. Bender and S. A. Orszag, Aduanced Mathematical Methods for Scientists and Engineers (McGraw-Hill, New York, 1978), p. 122.

${ }^{24}$ S. Hikami and E. Brezin, J. Phys. A 12, 759 (1979).

${ }^{25}$ D. Z. Goodson and D. R. Herschbach (to be published).

${ }^{26}$ J. G. Loeser and D. R. Herschbach, J. Phys. Chem. 89, 3444 (1985).

${ }^{27} \mathrm{~J}$. G. Loeser (to be published).

${ }^{28}$ D. Z. Goodson and D. R. Herschbach, J. Chem. Phys. 86, 4997 (1987).

${ }^{29}$ C. D. Lin, Adv. At. Mol. Phys. 22, 77 (1986). 